\title{
Optic neuropathy after repair of rhegmatogenous retinal detachment
}

\author{
Jonathan I. Huz ${ }^{1} \cdot$ Tian Xia $^{1} \cdot$ Larry Frohman $^{1} \cdot$ Roger E. Turbin ${ }^{1} \cdot$ Neelakshi Bhagat ${ }^{1}$
}

Received: 11 September 2018 / Accepted: 8 October 2018 / Published online: 1 November 2018

(c) The Royal College of Ophthalmologists 2018

The current report describes the intraoperative clinical setting and peri-operative variables of four patients who developed optic neuropathy after pars plana vitrectomy (PPV) for repair of primary rhegmatogenous retinal detachment (RRD). Baseline history, examination, and intraoperative parameters are displayed in Table 1. All patients underwent RRD repair under general anesthesia. Retrobulbar anesthesia was given through peri-bulbar approach using a blunt cannula at the beginning/end of the surgery.

Mean duration of surgery was $98.5 \mathrm{~min}$ (range 62-135 min). Oxygen saturation was $100 \%$ in all cases. No prior history of glaucoma or optic nerve disorder was elicited in any subject. Three eyes had silicone oil tamponade and one had C3F8. Figure 1 shows intraoperative OPP during the course of surgery. Of note, subject 2 experienced large MAP fluctuations resulting in low OPP $(<30 \mathrm{mmHg}$ for 10 -min and overall $<35 \mathrm{mmHg}$ for $30 \mathrm{~min}$ ). Subject 3 also had low OPP (30 $\mathrm{mmHg}$ for at least $10 \mathrm{~min}$ ). Maximum post-operative IOP noted in follow-up visits, before the diagnosis of optic neuropathy was made, ranged from 15 to $26 \mathrm{mmHg}$. Optic nerve pallor was first noted at an average of 49 days (range 6-74 days) after RRD repair surgery. No eyes had segmental or

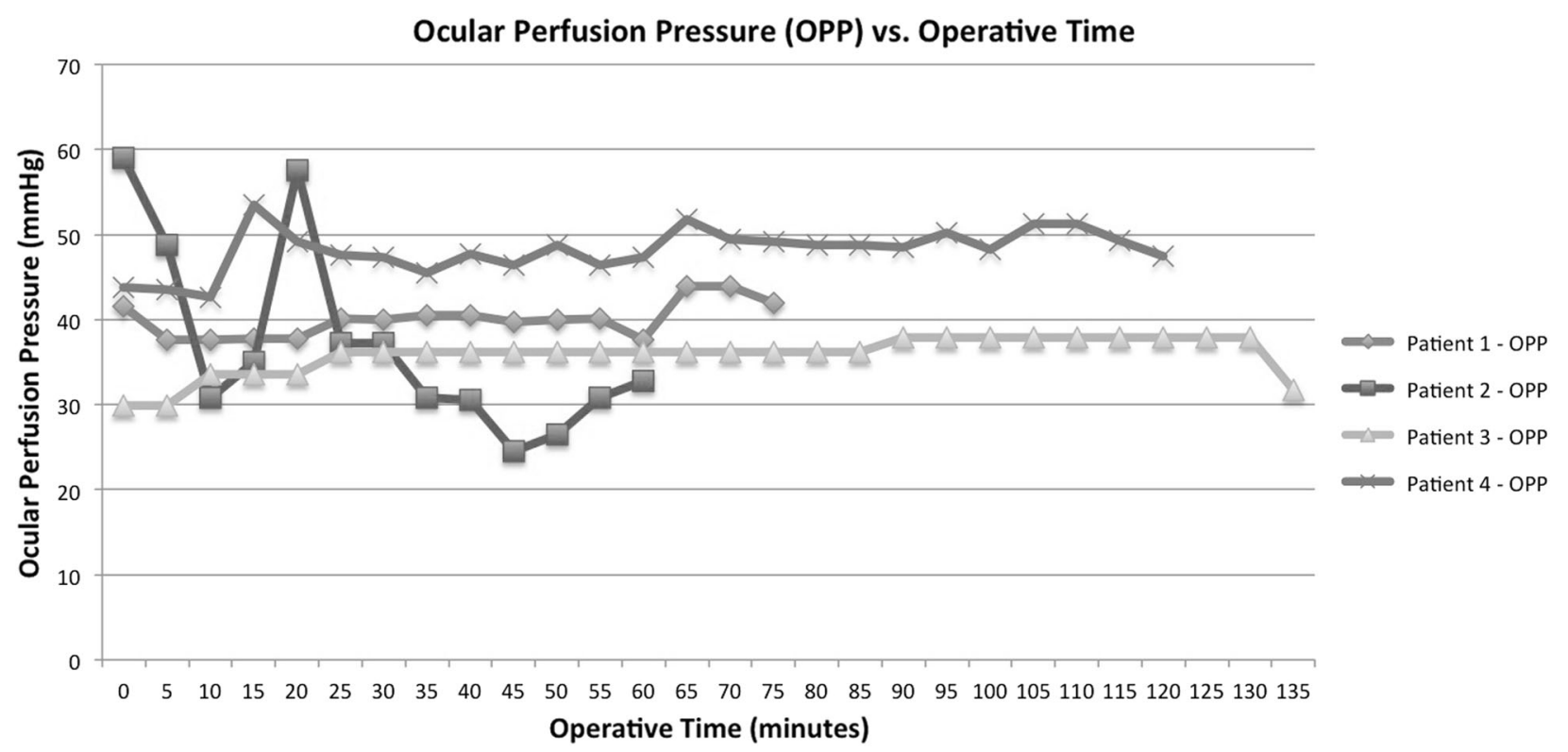

Fig. 1 Ocular perfusion pressure vs. operative time

Neelakshi Bhagat

bhagatne@njms.rutgers.edu

1 Institute of Ophthalmology and Visual Science, Rutgers-New Jersey Medical School, Newark, New Jersey, USA diffuse optic nerve edema, hyperemia or blurring of the disc margins on any post-operative visit. Extensive prothrombotic evaluation revealed a Factor $\mathrm{V}$ Leiden mutation, elevated sedimentation rate, and elevated anti-cardiolipin antibodies in subject 1 , hyperlipidemia in subject 2 , and hyperlipidemia and a slightly elevated $\mathrm{C} 4$ in subject 4 . 


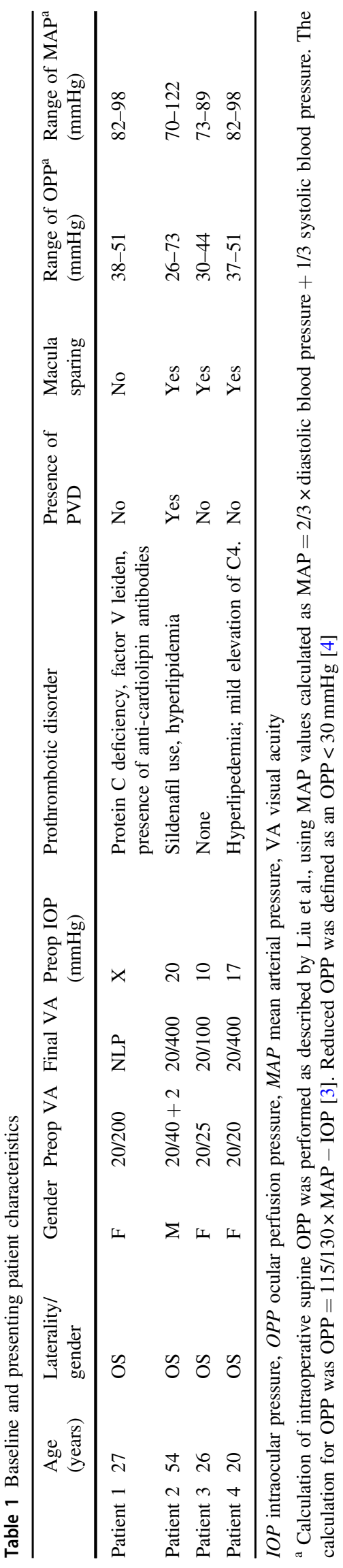

Peri-operative hypo-perfusion of optic nerve is a welldocumented phenomenon that can result in optic neuropathy [1]. Changes in mean blood pressure may have a greater effect on OPP and optic nerve head blood flow than fluctuations in IOP [1]. The optic nerve head can autoregulate its perfusion with fluctuation in blood pressure. Experiments with animal models have described that younger monkeys lose ability to autoregulate optic nerve perfusion once OPP falls below $30 \mathrm{mmHg}$ [1]; no comparable human studies have been performed but similar mechanisms may occur in young adults. In this study, large MAP fluctuations and reduced OPPs $(<30 \mathrm{mmHg})$ were seen in subjects 2 and 3 . Factor V Leiden mutation with elevated anti-cardiolipin antibodies in subject 1 and use of sildenafil [2] in subject 2 (detailed timeline of its use in relation to surgical repair could not be elicited) may have contributed in optic nerve perfusion. In the current study, the average age of patients was 32 years and the duration of operation ranged from 62 to $135 \mathrm{~min}$. Young patients do not have as robust compensatory response to reduced perfusion pressure as older individuals [1]. The longer duration of surgery with relatively prolonged reduced ocular perfusion could have contributed to the development of ischemic optic neuropathy.

Inadvertent optic nerve touch during air-fluid exchange can also cause optic nerve injury, however, there was no record of peripapillary hemorrhage or edema or sectoral pallor of the optic nerve in these eyes. This report highlights the need to be aware of possible effects of low OPP and large MAP variations in young individuals in intraocular procedures.

\section{Compliance with ethical standards}

Conflict of interest The authors declare that they have no conflict of interest.

\section{References}

1. Hayreh SS. Blood flow in the optic nerve head and factors that may influence it. Prog Retin Eye Res. 2001;20:595-624.

2. Karli SZ, Liao SD, Carey AR, Lam BL, Wester ST. Optic neuropathy associated with the use of over-the-counter sexual enhancement supplements. Clin Ophthalmol. 2014;8:2171-5.

3. Liu JH, Gokhale PA, Loving RT, Kripke DF, Weinreb RN. Laboratory assessment of diurnal and nocturnal ocular perfusion pressures in humans. J Ocul Pharmacol Ther. 2003;19:291-7.

4. Bansal AS, Hsu J, Garg SJ, Sivalingam A, Vander JF, Moster M, et al. Optic neuropathy after vitrectomy for retinal detachment: clinical features and analysis of risk factors. Ophthalmology. 2012;119:2364-70. 